\title{
Single-leg Standing Ability and Lower Limb Movement Analysis of Collegiate Footballers and Sedentary Students
}

Jiun Sien Lau, Vina Phei Sean Tan, Mohd Akmal Vahidi, Siti Hanisah Linggi Mohd Azam, Mohd Hafezi Mat Zain, Azilawati Azizan, Mazra Othman, Rosniwati Ghafar*

Exercise and Sports Science, School of Health Sciences, Universiti Sains Malaysia, Health Campus, 16150, Malaysia

\section{A R T I C L E I N F O}

Article history:

Received: 30 September, 2019

Accepted: 10 December, 2019

Online: 15 January, 2020

Keywords:

Balancing

Lower limbs

Footballer

Sedentary

\begin{abstract}
A B S T R A C T
Stability or balance is an integral component to perform daily activities without incurring injury or to be dependent on others. Sportsmen tend to have better balance than non-sports people but less is known about the single-leg balance ability. Furthermore, few studies analyzed the dynamic phase of single-leg stance that may contribute to better overall balance. Sports like football tend to have instances where the player's non-dominant leg keeps them in an upright position while the dominant leg kicks, passes and stops the ball. We aim to study the single-leg balance between collegiate footballers and sedentary students in eyes-closed (EC) and eyes-opened (EO) conditions and their contributing components to keep a body in an up-right position. Twenty collegiate footballers and 20 sedentary students conducted the unipedal stance test (UPST) on each leg with EO and EC conditions while standing on a force platform. We captured center of pressure (CoP) distance travelled, stance duration and using a 3D motion capture system, we assessed lower limb movement at six different anatomical sites. Results showed that footballers had better overall balance compared to sedentary students only in the non-dominant leg, EC condition with 12 footballers versus four sedentary students completing the full $45 \mathrm{~s}$ stance $(p=0.01)$ The other three UPST conditions did not show significant differences between groups. The CoP distance in the initial dynamic state and total UPST were both significantly shorter in footballers than sedentary students $(p<0.05)$ during the nondominant leg, EC stance. Our multivariable linear regression model significantly predicted time for UPST on non-dominant leg with EC up to $76.8 \%(p<0.001)$ with the first 5 -s of greater trochanter movement significantly contributing to total time taken for UPST in footballers. Overall, playing football may enhance balance control intrinsically especially for the non-dominant side while being less reliant on visual input to maintain balance.
\end{abstract}

\section{Introduction}

Balance is a crucial motor skill stemming from muscle synergies that lessens centre of pressure (CoP) displacement to effectively keep our body upright in a standing position, during locomotion and body orientation [1]. These muscle synergies are coordinated by the central nervous system with various inputs from our visual, vestibular and somatosensory systems [2]. In sports like gymnastics, balance is key to excel in the sport, however, other sports like football and basketball, static and dynamic balance are just as important although less apparent in their movements [3].

*Corresponding Author: Rosniwati Ghafar, rosnikk@usm.my
In football (considered as soccer in North America), most players shoot, pass and stop the ball with their dominant leg while the non-dominant leg supports their body weight [4]. Footballers often play and train on various turf conditions and wear cleated or non-cleated shoes. These conditions challenge trained footballers to develop better balance while mastering their football skills. Hence, top performing footballers have better posture stability and use less visual information to maintain their balance [5].

It is important to assess balance ability as it may relate to the level of sports performance and injury prevention. Single-limb and two-limb stance tests with eyes opened and with eyes closed are commonly used to test standing balance [6]. The unipedal stance 


\section{J.S. Lau et al. / Advances in Science, Technology and Engineering Systems Journal Vol. 5, No. 1, 01-07 (2020)}

test (UPST) is a common balance and standing ability assessment that can be easily performed and does not require additional equipment [7]. However, UPST lacks normative values for different age groups and balance ability changes with age [8]. Yet, UPST could detect changes of postural balance after 12 weeks of recreational soccer training and running in untrained men [9]. Researchers concluded that soccer training and high-intensity interval running enhanced the balance ability in their study participants [9]. As the UPST mainly measures the time taken to perform the test, we can also assess the center of pressure (CoP) during UPST using advanced technology to further understand the mechanisms of balance.

There are various types of force or pressure assessment systems to quantify CoP. Force platforms detect the ground reaction force and CoP. CoP can also be assessed from the force distribution across a sensor grid by other pressure assessment systems [10].

In sports biomechanics, motion data are used to study and observe human performance. The three-dimensional (3-D) motion capture system consists of infrared cameras with reflective markers attached to anatomical bony landmarks [11]. This system captures and measures motion that was hard to assess previously. 3-D motion capture plays an important role in sports to analyze physical limitations and for movement optimization [11]. Through the analyses, details about injury mechanism and movement will lead to new preventive methods and be used to improve a player's technique and performance [12].

There are two distinct phases in UPST based on Jonsson and colleagues' research [13]. The first phase is the first $5 \mathrm{~s}$ of UPST and also known as the dynamic phase where a rapid reduction in force variability can be seen. The better control a person has in the dynamic phase, the better their balance will be to complete the 45s UPST. The second phase is known as the static phase where the individual maintains a certain level of force variability that enables balance to be achieved [13]. Jonsson and colleagues concluded that the dynamic phase was key in assessing UPST [13]. Yet, we know little about lower limb influence on single-leg stance in young adults [14].

To our knowledge, studies that compared the balance ability between collegiate footballers and sedentary students are limited despite the possibility of recreational football play could enhance balance ability in previously untrained men [9]. Furthermore, balance is an important functional fitness component and should be cultivated to avoid balance-related injuries [15]. Therefore, our study aims to investigate if indeed simple football play could be related to better balance in young adults. We will compare the standing ability between collegiate footballers and sedentary students. We will also conduct further analysis on the dynamic phase of UPST to better understand lower limb control during the single-leg balance test.

\section{Methodology}

\subsection{Participants}

We recruited male collegiate students aged between 18 to 25 years old and grouped them as footballers $(n=20)$ or sedentary students $(n=20)$ based on our study's participant criteria. Footballers need to have a minimum of 2 years of football competition experience at collegiate level and/or exercise at least 3 times or more a week. Footballers need not be part of any specific training other than their recreational football games during noncompetition time. Sedentary students were those who exercised less than twice a week and do not participate in any sport competition. We excluded individuals with health conditions that may affect balance such as diabetes mellitus, musculoskeletal disorders, vestibular impairments and those with any lower limb injuries in the past 6 months. All participants provided informed consent and the Human Research Ethical Committee of Universiti Sains Malaysia provided ethical approval for this study.

\subsection{Study Procedure}

We measured participants' height $(\mathrm{cm})$ and weight $(\mathrm{kg})$ using a stadiometer and digital weight scale, respectively. Measures were done in duplicates and a third measure was obtained if difference between two measures were $0.4 \mathrm{~cm}$ in height or $0.2 \mathrm{~kg}$ in weight. We used the averaged value of measures obtained and calculated body mass index (BMI, $\mathrm{kg} / \mathrm{m}^{2}$ ) of all participants.

Participants conducted the UPST for both dominant and nondominant legs with i) eyes-opened (EO) and ii) eyes-closed (EC) conditions. Participants were instructed to kick a ball and the leg used was considered the dominant leg. Test was terminated early if participants i) used arms to balance their body, ii) touched the floor with the raised foot, iii) moved the standing foot to maintain their body posture, such as rotating the foot on the force platform or iv) opened their eyes in the EC condition. [8]. Each participant conducted three UPST trials and had $30 \mathrm{~s}$ of rest between trials. We recorded the total time taken to maintain the single-leg stance and stopped the UPST after $45 \mathrm{~s}$.

Participants stood on a force platform (Bertec, USA) and perform UPST by raising one leg as shown in Figure 1. The sampling rate of the platform was set at $120 \mathrm{~Hz}$. Participants stood barefooted on the level platform with the foot positioning marked on the force platform. This ensures uniformity and standardization of feet placement on the force platform which minimise variations between trials [16].

In the attempts to maintain posture in the UPST, body muscles are constantly adjusting and this creates 'sway' or body movement that can be captured by the force platform [17]. The force platform captures $\mathrm{CoP}$ in the $\mathrm{x}$ - and $\mathrm{y}$-axis in a constant flow and consequently, we assessed the distance travelled by CoP. Lesser distance and smaller CoP range usually indicates better balance skills [18].

A 3D motion capture system (Qualysis AB, Gothenburg Sweden) recorded lower limb movement and time during UPST. Six motion analysis cameras were placed surrounding the force platform as shown in Figure 2. In order to record movement of the limb during UPST, we attached 12 reflective markers to specific anatomical bony landmarks on the lower extremities. The reflective markers were attached to the greater trochanter, lateral side of the knee condyle, ankle and heel, and at the first and fifth metatarsal of both legs. The motion camera system captured the movement made during different UPST conditions and CoP from the force platform. Total distance was calculated from the movement of COP during performing UPST. The average time of the three trials and coordinates of $\mathrm{CoP}$ were extracted [8]. 
The first five seconds of UPST were further analysed to understand more about the dynamic phase of UPST. We used the results of the best trial out of the three attempts in this analysis. The best trial was selected based on the duration of the UPST and the shortest distance of CoP travelled during the same UPST trial.

\subsection{Statistical Analysis}

Statistical analyses were conducted using SPSS version 24.0 with significance set at $\mathrm{p}$-value $<0.05$. Time taken to complete UPST had a skewed distribution and we used Mann-Whitney to compare the differences between groups. We used independent ttest to compare the differences between footballers and sedentary students for the normally distributed data from different UPST conditions.

To understand the relation of lower limb movement on UPST, we conducted Pearson's correlation tests to identify the related anatomical sites during the dynamic phase that contributes to the time taken to complete UPST. Subsequently, we included relevant variables in a multivariable linear regression analysis.
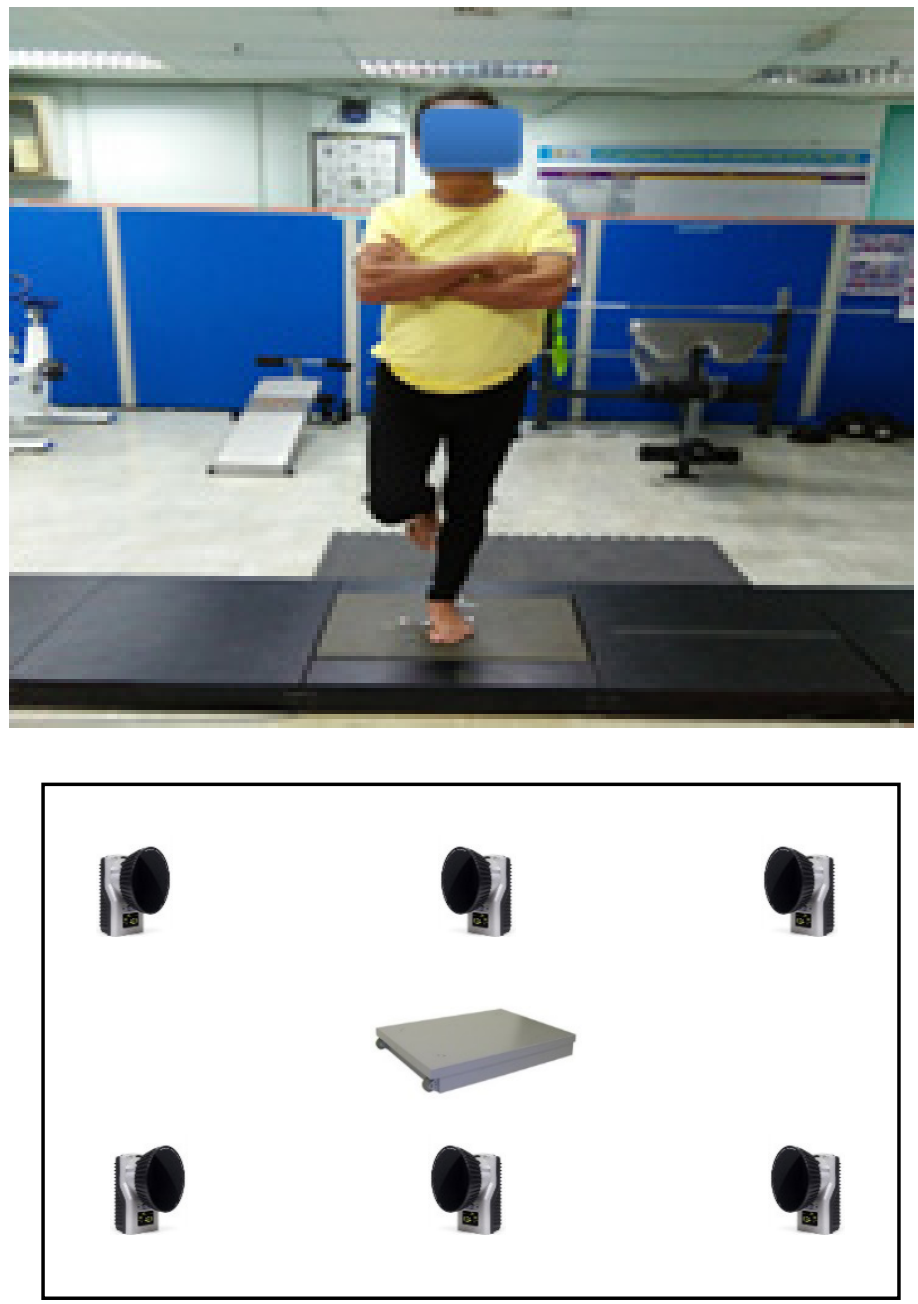

Figure 2: The field set-up of infrared cameras relative to the force platform

\section{Results}

Footballers were significantly younger than sedentary students by about 14 months but were similar in build (height, weight and BMI) as displayed in Table 1.
Table 1. Study participant characteristics in mean \pm standard deviation.

\begin{tabular}{lccc}
\hline & $\begin{array}{c}\text { Football } \\
(\mathbf{n}=\mathbf{2 0})\end{array}$ & $\begin{array}{c}\text { Sedentary } \\
(\mathbf{n}=\mathbf{2 0})\end{array}$ & $\boldsymbol{p}$-value \\
\hline Age $(\mathbf{y r})^{\dagger}$ & $21.4 \pm 1.1$ & $22.6 \pm 1.2$ & 0.002 \\
Height (m) & $1.69 \pm 0.07$ & $1.66 \pm 0.05$ & 0.089 \\
Weight (kg) & $66.3 \pm 14.0$ & $60.7 \pm 14.0$ & 0.056 \\
BMI (kg/m $\left.{ }^{2}\right)$ & $23.2 \pm 4.2$ & $22.3 \pm 5.8$ & 0.099 \\
\hline
\end{tabular}

${ }^{\dagger}$ Differences between groups analysed using independent t-test while other variables were analysed using Mann-Whitney test. $B M I$, body mass index.
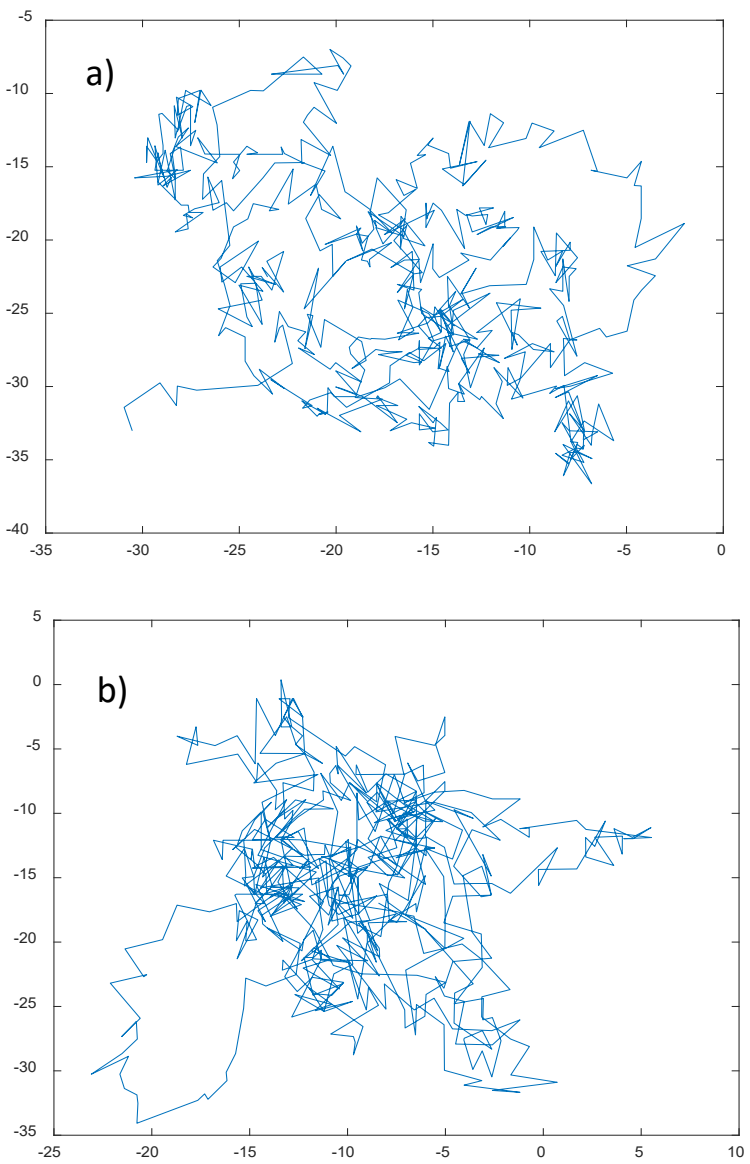

Figure 3: Examples of the 'best' or smallest range of $\mathrm{CoP}$ travel pathway for a) footballer and b) sedentary student during the non-dominant, eyes-closed condition.

\subsection{Time Taken to Perform UPST}

All footballers had no issues completing the UPST at the maximal $45 \mathrm{~s}$ when eyes were opened while standing on their dominant foot and only one person did not make the $45 \mathrm{~s}$ mark when standing on their non-dominant leg with EO (Table 2). Sedentary students did not perform any different than footballers on their dominant leg with EO or EC. However, there were three times more footballers $(n=12)$ that could complete UPST till $45 \mathrm{~s}$ compared to sedentary students $(n=4)$ when performing on their non-dominant leg with $\mathrm{EC}(\mathrm{p}=0.01)$.

As data was not normally distributed in the time taken to perform UPST in four different conditions, we did not detect any significant differences $(p>0.05)$ in time between groups as tested using the Mann-Whitney test. 


\subsection{Distance Travelled of CoP}

Footballers were no different in their total $\mathrm{CoP}$ distance travelled during UPST than sedentary students using their dominant leg in both EO and EC conditions. From Table 2, total CoP distance on non-dominant leg, EO UPST were no different between groups too $(\mathrm{p}>0.05)$. In non-dominant leg, EC UPST, footballers significantly swayed less compared to sedentary students $(\mathrm{p}=0.01)$.

\subsection{Distance Travelled of CoP in Dynamic Phase}

Examples of CoP patterns of a footballer versus a sedentary student are presented in Figure 3 and 4 during the dynamic phase or the first 5-s portion of UPST. We selected the best (smallest) and worst (largest) range of $\mathrm{CoP}$ for both groups respectively in the non-dominant leg, EC session. Footballer CoPs showed less complexity of pattern within the range compared to a sedentary students' CoP travel path.

The 5-s CoP distance travelled were thus significantly different $(p=0.021)$ between footballers and sedentary students when analysed using Mann-Whitney test (Table 2). The rest of the conditions were no different in 5-s CoP distance reported between footballers and sedentary students.
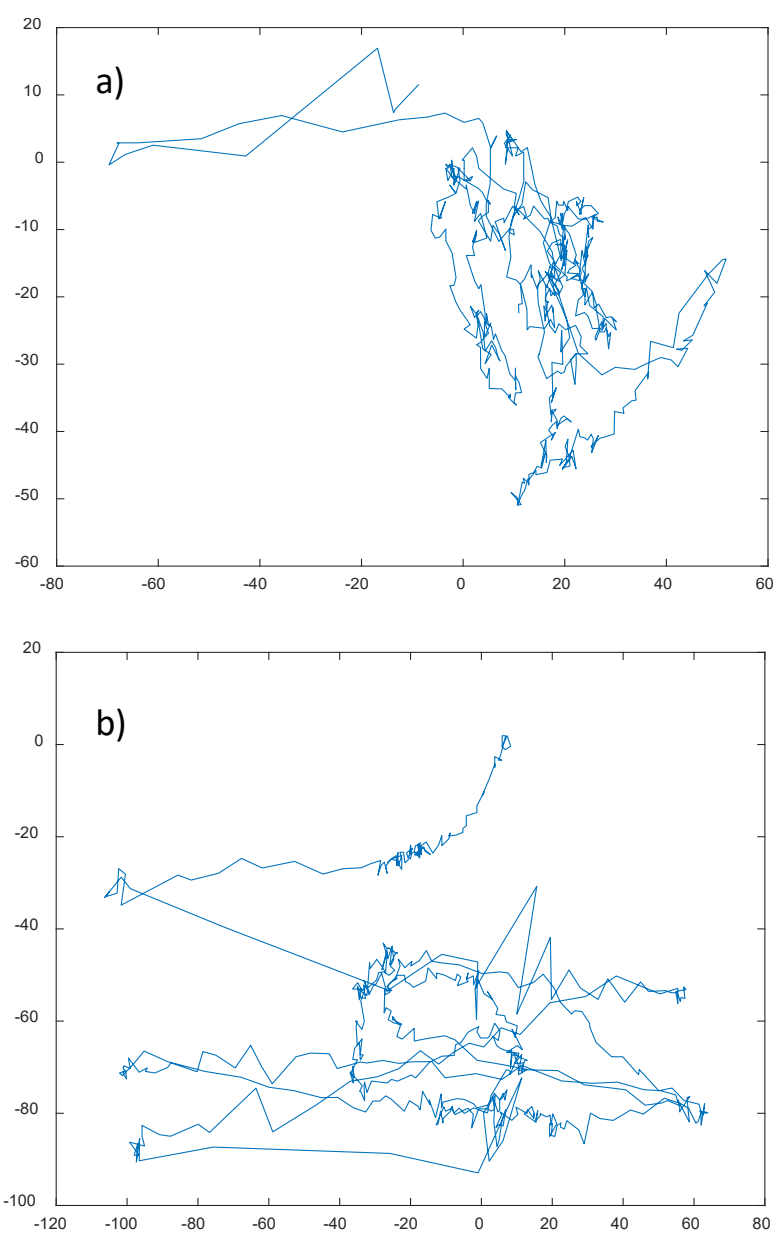

Figure 4: Example of a 'worst' or largest range of $\mathrm{CoP}$ travel pathway for a) footballer and b) sedentary student during the non-dominant, eyes-closed condition.

\subsection{Non-dominant Leg, EC UPST Time and Lower Limb Control During Dynamic Phase}

In Table 3, we used the best trial to further study the contribution of lower limb movement to overall time taken to complete UPST. Footballers could maintain their UPST significantly longer than sedentary students using the nondominant leg in the EC condition.

We analysed the first 5-s of lower limb function on single-leg balance during the dynamic phase toward overall time taken to complete UPST. Interestingly, the lower limb movement in all six anatomical sites were not significantly different between footballers and sedentary students (Table 3). Yet, we observed significant negative correlation of all lower limb movement sites to time taken to complete UPST (Table 4). However, we had to remove four data sets due to noise in lower limb movement analysis.

Subsequently, we included the lower limb movements in the first 5-s of UPST to predict time to complete UPST. We analysed footballers and sedentary students separately. We found that different sites of lower limb movement were not predictive of overall time to complete UPST in sedentary students $(\mathrm{p}>0.05)$. However, our regression model in footballers (Table 5) showed that the overall model significantly predicted up to $76.1 \%$ $(p<0.001)$ of total time to complete UPST. In that regression model, although we have ankle, knee and greater trochanter as predictor variable, the movement from the greater trochanter was the significant variable to predict time to complete UPST. The first and fifth metatarsal and heel sites were removed due to high correlation within the regression model (variance inflation factor $>5)$.

\section{Discussion}

The objective of the study is to compare the standing balance ability between male collegiate football players and sedentary students and to understand the influence in the dynamic phase toward overall single-leg standing. We found that footballers on non-dominant leg with eyes-closed (EC) could significantly balance better than sedentary students as they completed more trials, had a longer balance time and $\mathrm{CoP}$ distance was shorter in the dynamic and static phase. Furthermore, we found that the footballers' greater trochanter control in the early dynamic phase significantly predicts overall balance performance in the nondominant leg, EC condition. Whereas lower limb movements of sedentary students may not be helpful in maintaining balance during an EC, single-leg stand.

Differences in balance ability between sports/trained with untrained individuals are known. Specifically, in football, two other studies have showed that the highly skilled footballers relied less on visual information to maintain a stable posture [5,19]. Naturally, footballers were also known to be proficient in using their non-dominant leg as well as their dominant side [20]. Studies of other sports that included gymnastics and swimming also showed better postural control and efficient use of somatosensory and otolithic input to maintain body posture [21, 22]. Results of sports contributing to better balance is even apparent in recreational players whereby simple football training resulted in adapted visual, somatosensory and vestibular senses that improved 


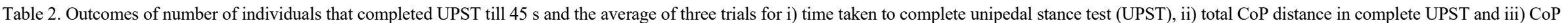
distance in the first 5 -sec of UPST in footballers and sedentary students, reported as median (inter-quartile range).

\begin{tabular}{|c|c|c|c|c|c|c|c|c|}
\hline & \multicolumn{4}{|c|}{ Footballer $(\mathrm{n}=\mathbf{2 0})$} & \multicolumn{4}{|c|}{ Sedentary students $(\mathrm{n}=\mathbf{2 0})$} \\
\hline & \multicolumn{2}{|c|}{ Dominant leg } & \multicolumn{2}{|c|}{ Non-dominant leg } & \multicolumn{2}{|c|}{ Dominant leg } & \multicolumn{2}{|c|}{ Non-dominant leg } \\
\hline & EO & EC & EO & $\mathbf{E C}$ & EO & EC & EO & $\mathbf{E C}$ \\
\hline Completed max. 45-s UPST (n) & 20 & 11 & 19 & 12 & 19 & 9 & 18 & $4 *$ \\
\hline Duration of UPST (s) & $45.0(0.0)$ & $23.0(32.6)$ & $45.0(0.0)$ & $31.2(27.1)$ & $45.0(0.0)$ & $20.4(21.6)$ & $45.0(13.7)$ & $24.4(21.8)$ \\
\hline Total CoP distance $(\mathrm{mm})^{\dagger}$ & $144.8(2.6)$ & $140.1(3.9)$ & $144.3(3.0)$ & $140.5(3.9)$ & $145.8(0.6)$ & $140.8(5.0)$ & $145.8(0.6)$ & $144.1(4.7)^{*}$ \\
\hline CoP distance in first 5-s (mm) & $133.8(0.1)$ & $134.0(0.7)$ & $133.8(0.1)$ & $134.3(0.5)$ & $133.8(0.1)$ & $134.1(0.3)$ & $133.8(0.1)$ & $134.0(0.3)^{*}$ \\
\hline
\end{tabular}

EO, eyes-opened; EC, eyes-closed.

*Significant differences between footballers and sedentary students in similar leg and eye conditions with $p$-value $<0.05$ as analysed by Mann-Whitney test or independent t-test.

Differences between groups were analysed using independent $t$-test and results reported as mean and standard deviation.

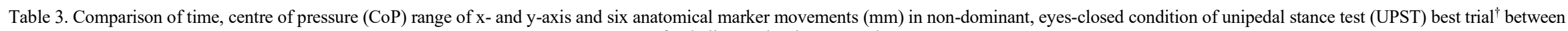
footballers and sedentary students.

\begin{tabular}{|c|c|c|c|}
\hline & \multicolumn{2}{|c|}{ Median (IQR) } & \multirow[b]{2}{*}{$p$-value } \\
\hline & Football $(n=20)$ & Sedentary $(n=20)$ & \\
\hline \multicolumn{4}{|l|}{ Complete UPST } \\
\hline Time (s) & $45.0(21.2)$ & $29.4(29.2)$ & $0.044^{*}$ \\
\hline CoP $x$-axis range $(\mathbf{m m})$ & $45.4(19.0)$ & $41.3(20.2)$ & 0.646 \\
\hline CoP y-axis range $(\mathrm{mm})$ & $41.4(6.4)$ & $42.1(12.9)$ & 0.626 \\
\hline \multicolumn{4}{|c|}{ Distance travelled in first 5-s of UPST } \\
\hline $1^{\text {st }}$ metatarsal $(\mathrm{mm})$ & $100.6(73.4)$ & $103.5(65.9)$ & 0.534 \\
\hline $5^{\text {th }}$ metatarsal $(\mathrm{mm})$ & $73.6(40.1)$ & $72.1(54.6)$ & 0.787 \\
\hline Ankle (mm) & $64.5(44.8)$ & $61.5(30.4)$ & 0.607 \\
\hline Heel (mm) & $43.4(26.3)$ & $55.8(18.7)$ & 0.162 \\
\hline Knee (mm) & $146.1(107.7)$ & $142.2(87.5)$ & 0.978 \\
\hline Greater trochanter (mm) & $118.0(68.7)$ & $134.4(54.6)$ & 0.555 \\
\hline
\end{tabular}

${ }^{\dagger}$ Best trial is defined as the longest time taken and with the shortest CoP distance travelled during UPST. $I Q R$, inter-quartile range. 
Table 4: Correlation between time for complete UPST time (s) and lower limb maker distances $(\mathrm{mm})$ during the dynamic phase $(\mathrm{n}=36)$.

\begin{tabular}{lcc}
\hline \multicolumn{1}{c}{ Lower limb sites } & \multicolumn{2}{c}{ Time } \\
\hline First metatarsal & -0.50 & $\boldsymbol{p}$-value \\
Fifth metatarsal & -0.46 & 0.001 \\
Ankle & -0.38 & 0.003 \\
Knee & -0.44 & 0.012 \\
Heel & -0.55 & 0.004 \\
Greater trochanter & -0.61 & $<0.001$ \\
\hline
\end{tabular}

Table 5: Multivariable linear regression model to predict the influence of greater trochanter, knee and ankle on time taken to complete unipedal stance test in

\begin{tabular}{lllllc}
\multicolumn{7}{c}{ collegiate footballers $(\mathrm{n}=18)}$. \\
\hline Variables & $\boldsymbol{\beta}$ & $\mathbf{B}$ & SE & $\boldsymbol{p}$ & $\mathbf{9 5 \%}$ CI \\
\hline Greater & -0.77 & -0.22 & 0.06 & 0.002 & $-0.35,-0.09$ \\
Krochanter & & & & & \\
Ankee & -0.09 & -0.02 & 0.04 & 0.644 & $-0.09,0.06$ \\
Constant & -0.06 & -0.02 & 0.05 & 0.685 & $-0.13,0.09$ \\
\hline - & 69.1 & 5.2 & $<0.001$ & - \\
\hline
\end{tabular}

$\beta$, standardized coefficient; $B$, unstandardized coefficient; SE, coefficients standard error; CI, confidence interval

posture and neuromuscular control [9]. Thus, engaging in recreational sports, may it be football or other sports, may have added advantage to balance skills even in young adults. This may be a good foundation where balance is enhanced and hopefully maintained to prevent falls and fractures in later life.

The better balance performance in footballers compared to sedentary students were apparent in the sway observed, which was measured as the CoP distance travelled. Regardless if it was during the early dynamic phase (first 5-s) or static phase (after 5 s), footballers had less sway and were able to keep their movement to a minimal throughout the UPST on their non-dominant leg with eyes closed. The CoP distance was significantly shorter despite the range of $\mathrm{CoP}$ values in both the $\mathrm{x}$ - and $\mathrm{y}$-axis being similar in footballers and sedentary students. Thompson and colleagues found out that footballers have better control in their anteriorposterior and mediolateral stability than the non-athletes [23]. This finding is in line with our outcome of this study.

Lastly, footballer have their hips to thank for keeping their UPST balance time longer compared to sedentary students. Their greater trochanter movement could significantly predict up to $77 \%$ of the time to complete the UPST while using their non-dominant leg and without visual input. This means participation in recreational football may indirectly provide players with better balance and postural control from the hip that may be missing in more sedentary people. As the hip is a known controller of postural stability [24], having specific and targeted exercises to improve hip control alone for balance may be difficult. Yet, participating in football games up to three times a week could train our balance intrinsically while having fun. Krustrup and colleagues mentioned that the training component of football were able to improve the postural balance of the players [25].

\section{Conclusion}

This is a cross-sectional study and our results are inferential at best. Our study sample size could have been improved as well to obtain a better normal distribution of data. However, non- parametric statistical analyses are equally powerful to parametric tests when used appropriately [26].

To improve on our balance protocol, we suggest future studies consider incorporating a warm-up component before performing UPST as it may improve overall balance [27]. With regards to the maximum time of $45 \mathrm{~s}$, this may be suitable for young adults but researchers may consider different times for different type of population or even consider not having a time limit to include the possibility of muscle fatigue in different types of population.

The strength of our study highlights the need to understand muscle control towards balance in early dynamic phases that will contribute to the completion of the balance task at hand. Subsequently, use of advanced technology such as a force platform to assess $\mathrm{CoP}$ are ideal and provides a better understanding of the intricacies that are working to provide posture and stability in individuals of all ages.

Further studies are needed to support our current findings and intervention studies in sedentary young adults will be beneficial to inform in this area. More work can be done to understand the contribution of lower limb muscles and activation especially in the more vulnerable groups like older adults and people with osteoporosis to prevent falls and fractures.

\section{Conflict of Interest}

There is no conflict of interest reported between the authors.

\section{Acknowledgment}

This project was partly funded by USM Short Term Grant (304/PPSK/61312135).

\section{References}

[1] J. Winearls, "Posture: its function in efficient use of the human organism as a total concept" Agressologie, 13, 99-101, 1972.

[2] R. Lepres, A. X. Bigard, J. P. Diard, J. F. Gouteyron, C. Y. Guezennec, "Posture control after prolonged exercise" Eur J Appl Physiol Occup Physiol.. 76(1), 55-61, 1997. https://doi.org/10.1007/s004210050212

[3] P. G. Gerbino, E. D. Griffin, D. Zurakowski, "Comparison of standing balance between female collegiate dancers and soccer players" Gait Posture, 26(4), 501-507, 2007. doi:10.1016/j.gaitpost.2006.11.205

[4] D. A. Winter, A. E. Patla, J. S. Frank, "Assessment of balance control in humans". Med Prog Technol, 16(1-2), 31-51, 1990.

[5] T. Paillard, F. Noe, "Effect of expertise and visual contribution on postural control in soccer" Scand J Med Sci Sports, 16(5), 345-348, 2006. DOI: $10.1111 / \mathrm{j} .1600-0838.2005 .00502 . \mathrm{x}$

[6] J. Jerosch, L. Thorwesten, H. Bork, M. Bischof, "Is prophylactic bracing of the ankle cost effective?" Orthopedics, 19(5), 405-414, 1996. https://doi.org/10.3928/0147-7447-19960501-10

[7] B. L. Riemann, R. Schmitz, "The relationship between various modes of single leg postural control assessment" Int. J. Sports Phys. Ther., 7(3), 257266, 2012.

[8] B .A. Springer, R. Marin, T. Cyhan, H. Roberts, N. W. Gill, "Normative values for the unipedal stance test with eyes open and closed" J Geriatr Phys Ther, 30(1), 8-15, 2007

[9] M. D. Jakobsen, E. Sundstrup, P. Krustrup, P. Aagaard, "The effect of recreational soccer training and running on postural balance in untrained men" Eur J Appl Physiol, 111(3), 521-530, 2011.DOI 10.1007/s00421-010$1669-2$

[10] E. S. Chumanov, C. D. Remy, D. G. Thelen, D. G, "Computational techniques for using insole pressure sensors to analyse three-dimensional joint kinetics" Comput Method Biomec, 13(5), 505-514, 2010. DOI: $10.1080 / 10255840903350148$

[11] B. Pueo, J. M. Jimenez-Olmedo, "Application of motion capture technology for sport performance analysis” Retos, 32, 241-247, 2017. 
[12] S. L. Colyer, M. Evans, D. P. Cosker, A. I. Salo, "A review of the evolution of vision-based motion analysis and the integration of advanced computer vision methods towards developing a markerless system" Sports MedOpen, 4(1), 24, 2018. https://doi.org/10.1186/s40798-018-0139-y

[13] E. Jonsson, A. Seiger, H. Hirschfeld, "One-leg stance in healthy young and elderly adults: a measure of postural steadiness?" Clin Biomech 19, 21(3), 688-694, 2004. doi:10.1016/j.clinbiomech.2004.04.002

[14] T. Muehlbauer, C. Mettler, R. Roth, U. Granacher, "One-leg standing performance and muscle activity: are there limb differences?" J Appl Biomech, 30(3), 407-414, 2014. https://doi.org/10.1123/jab.2013-0230

[15] N. Takeshima, N. L. Rogers, M. E. Rogers, M. M. Islam, D. Koizumi, S. Lee, "Functional fitness gain varies in older adults depending on exercise mode" Med Sci Sports Exerc, 39(11), 2036-2043, 2007. DOI: $10.1249 / \mathrm{mss} .0 \mathrm{~b} 013 \mathrm{e} 31814844 \mathrm{~b} 7$

[16] J. A. Kim, O. B. Lim, C. H, Yi, "Difference in static and dynamic stability between flexible flatfeet and neutral feet" Gait posture, 41(2), 546-550, 2015. https://doi.org/10.1016/j.gaitpost.2014.12.012

[17] A. Karlsson, G. Frykberg, "Correlations between force plate measures for assessment of balance" Clin Biomech, 15(5), 365-369, 2000. doi:10.1016/s0268-0033(99)00096-0

[18] A. Mansfield, E. L. Inness, "Force plate assessment of quiet standing balance control: Perspectives on clinical application within stroke rehabilitation" Rehabilitation Process and Outcome, 4, 7-5, 2015. doi:10.4137/RPO.S20363.

[19] T. Paillard, F. Noe, T. Riviere, V. Marion, R. Montoya, P. Dupui, "Postural performance and strategy in the unipedal stance of soccer players at different levels of competition" J. Athl. Train., 41(2), 172-176, 2006.

[20] R. Barone, F. Macaluso, M. Traina, V. Leonardi, F. Farina, V. Felice, "Soccer players have a better standing balance in nondominant one-legged stance" Open Access J Sports Med, 2, 1-6, 2011. doi:10.2147/OAJSM.S12593

[21] C. D. Davlin, "Dynamic balance in high level athletes" Percept Mot Skills, 98(3_suppl), 1171-1176, 2004.

[22] L. Bringoux, L. Marin, V. Nougier, P. A. Barraud, C. Raphel, "Effects of gymnastics expertise on the perception of body orientation in the pitch dimension" J Vestib Res, 10(6), 251-258, 2000.

[23] L. Thompson, M. Badache, S. Cale, L. Behera, N. Zhang, "Balance performance as observed by center-of-pressure parameter characteristics in male soccer athletes and non-athletes" Sports, 5(4), 86, 2017. doi:10.3390/sports5040086

[24] L. Allet, H. Kim, J. Ashton-Miller, T. De Mott, J. K. Richardson, "Frontal plane hip and ankle sensorimotor function, not age, predicts unipedal stance time" Muscle Nerve, 45(4), 578-585, 2012. DOI 10.1002/mus.22325

[25] P. Krustrup, P. Aagaard, L. Nybo, J. Petersen, M. Mohr, J. Bangsbo, "Recreational football as a health promoting activity: a topical review" Scand J Med Sci Sports, 20(Suppl. 1), 1-13, 2010. doi: 10.1111/j.16000838.2010.01108.x

[26] M. Hughes, S. M. Cooper, A. Nevill, "Analysis procedures for nonparametric data from performance analysis" Int J Perf Anal Spor., 2(1), 620, 2002. doi:10.1080/24748668.2002.11868257

[27] A. Daneshjoo, A. H. Mokhtar, N. Rahnama, A. Yusof, "The Effects of Comprehensive Warm-Up Programs on Proprioception, Static and Dynamic Balance on Male Soccer Player". PLoS ONE, 7(12): e51568, (2012) doi:10.1371/journal.pone. 0051568 